\title{
Acute Paraspinal Compartment Syndrome in an Unconscious Patient
}

\author{
Talha Ahmed ${ }^{1}$, Ayesha Safdar ${ }^{2}$, Tamoor Ahmed ${ }^{3}$, Talal Ahmad ${ }^{4}$, Lia Losonczy ${ }^{5}$ \\ 1. Internal Medicine, University of Maryland Medical Center, Baltimore, USA 2. Internal Medicine, Army Medical \\ College, Rawalpindi, PAK 3. Internal Medicine, King Edward Medical University/Mayo Hospital, Lahore, PAK 4. Internal \\ Medicine, Services Hospital, Lahore, PAK 5. Emergency Medicine, The George Washington University Medical Center, \\ Washington DC, USA
}

Corresponding author: Talha Ahmed, atalha858@yahoo.com

\begin{abstract}
Compartment syndrome can be a limb-threatening emergency that may require immediate intervention. It usually involves the extremities but any closed compartment of the body is susceptible to it. Paraspinal compartment extends on both sides of the spine. Prolonged lying on the back in unconscious patients leads to muscle edema which eventually leads to increase pressure in the compartment. Neurovascular comprise is a dreaded complication of compartment syndrome. Paraspinal compartment is a potential site of compartment syndrome particularly in unconscious patients and it requires prompt diagnosis, careful monitoring, immediate medical attention and even warranting surgical intervention in certain cases.
\end{abstract}

Categories: Emergency Medicine, Internal Medicine, Trauma

Keywords: compartment syndrome, paraspinal compartment syndrome, substance abuse, seizure, unconscious

\section{Introduction}

Compartment syndrome can be a limb-threatening emergency that may require immediate intervention. It can involve any closed compartment of the body with the most common site being the anterior distal lower extremity or the anterior compartment of the leg [1]. We describe a case of paraspinal compartment syndrome in an unconscious patient, secondary to polysubstance abuse and seizures. Prolonged periods of pressure on the compartments of the back particularly the paraspinal compartment can lead to paraspinal compartment syndrome which can be easily missed in an unconscious patient. This requires performing a thorough clinical examination particularly in patients with unexplained elevations in creatine kinase (CK) levels that are not being cleared from the body. Appropriate and timely intervention can prove to be life saving [2].

Received 03/02/2020 Review began 03/04/2020 Review ended 03/07/2020 Published 03/09/2020

○) Copyright 2020 Ahmed et al. This is an open access article distributed under the terms of the Creative Commons Attribution License CC-BY 4.0., which permits unrestricted use, distribution, and reproduction in any medium, provided the original author and source are credited.

\section{Case Presentation}

A 49-year-old male with a past history of seizures on antiepileptics was brought to the hospital (due to confusion and altered mental status) after his wife called the emergency medical service (EMS). The patient had a reported episode of seizure on arrival to the EMS after which he continued to remain confused and somnolent. The patient had stable vital signs on presentation with a heart rate of 65 beats per minute, blood pressure of 130/78 $\mathrm{mmHg}$, a body mass index (BMI) of $26 \mathrm{~kg} / \mathrm{m} 2$ and was afebrile with oxygen saturation of $95 \%$ on room air. Urine toxicology screen in the emergency department (ED) was positive for cocaine and the urine fentanyl dip test was positive. Computed tomography (CT) of the head was normal. Creatine kinase (CK) was elevated to 4550 units/L (normal range: 22-198 units/L) at the time of admission. Other significant lab findings included mild transaminitis with aspartate aminotransferase (AST) of 148 units/L (normal range: 10-40 units/L) and alanine aminotransferase (ALT) of 90 units/L (normal range: 7-56 units/L). The patient was admitted to the intensive care unit (ICU), was given a bolus of three liters fluids, and continued on maintenance fluids to produce $200 \mathrm{ml}$ of urine per hour. Serial measurements of CK did not show significant improvement despite fluid resuscitation and stayed at 4530 units/L even after 24 hours of significant hydration. This prompted a complete re-examination of the body to evaluate for compartment syndrome which revealed significant swelling on the left paraspinal area in the lower thoracic and lumbar region extending laterally along the external oblique muscle. As the illicit drugs started clearing from the patient's body, his mental status improved and he was able to complain of pain and tenderness to palpation at the swollen site. CT scan of the abdomen and pelvis, as well as the lumbar and thoracic spine, were ordered for evaluation of the swelling. There was asymmetry and edema along the left paraspinal muscles especially the external oblique (Figure 1). 


\section{Cureus}

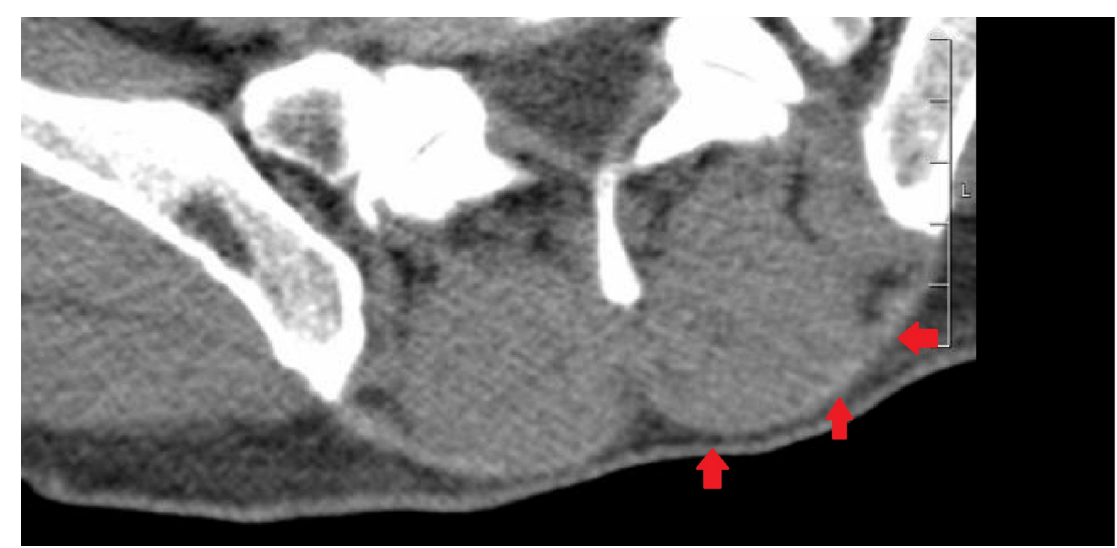

FIGURE 1: Computed tomography scan of the lumbar spine showing swelling of the left paraspinal compartment

The patient was re-positioned to decrease compression on the affected site while continuing fluid resuscitation and general surgery was consulted for possible intervention, if needed. It was decided to do conservative management initially with frequent clinical examinations, serial CK level monitoring. and repositioning of the patient. Repeat CK after 36 hours of being in the ICU started trending down with the leftsided paraspinal area showing decreased swelling, pain, and tenderness; hence, it did not warrant measuring the tissue pressures. Fortunately, the patient's renal function remained intact.

\section{Discussion}

Paraspinal compartment syndrome is a rare entity that requires a high index of suspicion for diagnosis. This syndrome can be classified into acute or chronic types. Acute paraspinal compartment syndrome subtypes include the following: atraumatic (due to downhill skiing, surf boarding, weight lifting), direct trauma (accidents, prolonged pressure, or direct injury) and secondary to non-spinal surgery (due to aortic bypass, gastric bypass) [3]. This patient presented with acute paraspinal compartment syndrome from prolonged pressure on the affected compartment from unconsciously lying down on the back for a long period of time due to substance abuse and seizures.

Unlike extremity compartment syndrome where classical signs include pain, paresthesias, paresis, pallor, poikilothermia, and pulselessness, in acute paraspinal compartment syndrome, all these symptoms are not appreciated [4]. Typical clinical presentation includes localized pain, tenderness, rigidity, and paresthesia [5]. Interestingly, patients may also have decreased or absent bowel sounds and activities that raise the intraabdominal pressure which may exacerbate symptoms [6].

Laboratory findings supporting the diagnosis include elevated CK, urine myoglobin, creatinine, AST, and ALT [7]. Edema, inflammation, and/or hematoma on magnetic resonance imaging (MRI) usually identifies the paraspinal compartment $[8,9]$. Measuring of intra-compartment pressures confirms the diagnosis with pressures that are usually significantly elevated $(70-80 \mathrm{mmHg})[10]$.

Once acute paraspinal compartment syndrome is suspected, management should focus on the following: aggressive resuscitation with intravenous fluids, avoidance of nephrotoxic drugs, cessation of any further traumatic activities, and urgent surgical decompression with fasciotomy. Although conservative management with non-operative intervention is an option, patients have demonstrated improved outcomes with surgical fasciotomy even when it is delayed [11].

In our case, conservative management with very close monitoring and aggressive resuscitation yielded favorable outcomes. This case, however, demonstrates that in unconscious patients with persistently elevated CK, we should be extremely vigilant with our clinical examination to evaluate all the compartments of the body particularly the paraspinal compartment.

This article was presented as a poster. (Poster: Talha Ahmed, Ayesha Safdar, Lia Losonczy. Acute Paraspinal Compartment Syndrome Secondary to Polysubstance Abuse and Seizures. CHEST Annual Meeting; Oct 2019).

\section{Conclusions}

Paraspinal compartment syndrome can be acute secondary to weight lifting, skiing, surfing, direct trauma, non-spinal

surgery including aortic, and gastric bypass or chronic secondary to chronic exertion. Conservative measures 
are the first line of management and include opioids for pain control and treatment of rhabdomyolysis. Although rare, it is prudent to consider the paraspinal area as a potential site for acute compartment syndrome in unconscious patients with a history of substance abuse and significantly elevated CK. By providing appropriate interventions, potentially serious consequences can be avoided.

\section{Appendices}

The abstract of this case report was presented as a poster at the 'CHEST' conference in 2019.

\section{Additional Information \\ Disclosures}

Human subjects: Consent was obtained by all participants in this study. Conflicts of interest: In compliance with the ICMJE uniform disclosure form, all authors declare the following: Payment/services info: All authors have declared that no financial support was received from any organization for the submitted work. Financial relationships: All authors have declared that they have no financial relationships at present or within the previous three years with any organizations that might have an interest in the submitted work. Other relationships: All authors have declared that there are no other relationships or activities that could appear to have influenced the submitted work.

\section{References}

1. Nathan ST, Roberts CS, Deliberato D: Lumbar paraspinal compartment syndrome. Int Orthop. 2012, 36:1221-7. 10.1007/s00264-011-1386-4

2. Allerton C, Gawthrope IC: Acute paraspinal compartment syndrome as an unusual cause of severe low back pain. Emerg Med Australas. 2012, 24:457-9. 10.1111/j.1742-6723.2012.01584.x

3. Calvert N, Bhalla T, Twerenbold R: Acute exertional paraspinal compartment syndrome. ANZ J Surg. 2012, 82:564-5. 10.1111/j.1445-2197.2012.06117.x

4. Karam MD, Amendola A, Lattes MS: Case report: successful treatment of acute exertional paraspinal compartment syndrome with hyperbaric oxygen therapy. Iowa Orthop J. 2010, 30:188-90.

5. Levine M, Levitan R, Skolnik A: Compartment syndrome after "bath salts" use: a case series . Ann Emerg Med. 2013, 61:480-483. 10.1016/j.annemergmed.2012.11.021

6. Paryavi E, Jobin CM, Ludwig SC, Zahiri H, Cushman J: Acute exertional lumbar paraspinal compartment syndrome. Spine. 2010, 35:1529-33. 10.1097/BRS.0b013e3181ec4023

7. Minnema BJ, Neligan PC, Quraishi NA, Fehlings MG, Prakash S: A case of occult compartment syndrome and nonresolving rhabdomyolysis. J Gen Intern Med. 2008, 23:871-4. 10.1007/s11606-008-0569-1

8. Ahmed T. Safdar A, Losonczy L: Acute paraspinal compartment syndrome secondary to polysubstance abuse and seizures. Chest. 2019, 156:A2136.

9. Haig AJ, Hartigan AG, Quint D: Low back pain after nonspinal surgery: the characteristics of presumed lumbar paraspinal compartment syndrome. PMR. 2009, 1:383-8. 10.1007/s11606-008-0569-1

10. Khan RJ, Fick DP, Guier CA, Menolascino MJ, Neal MC: Acute paraspinal compartment syndrome. J Bone Joint Surg Am. 2005, 87:1126-8. 10.2106/JBJS.D.02133

11. Rha EY, Kim DH, Yoo G: Acute exertional lumbar paraspinal compartment syndrome treated with fasciotomy and dermatotraction: case report. J Plast Reconstr Aesthet Surg. 2014, 67:425-426. 10.1016/j.bjps.2013.10.026 